
\title{
$\begin{array}{ll}\text { Research Square } & \begin{array}{l}\text { Preprints are preliminary reports that have not undergone peer review. } \\ \text { They should not be considered conclusive, used to inform clinical practice, } \\ \text { or referenced by the media as validated information. }\end{array}\end{array}$
}

\section{Isolation of Antibiotic-Resistant Bacteria From The Atmospheric Air In Hospital Wards And Outdoor Areas In Kuwait During Sandstorms}

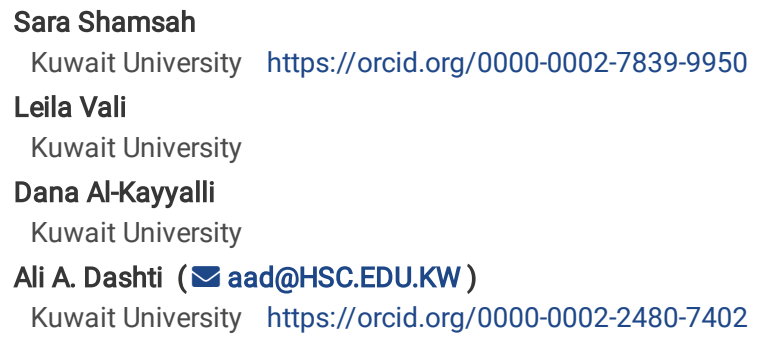

\section{Research article}

Keywords: Airborne bacteria, Antibiotic resistance, Clinical settings.

Posted Date: November 12th, 2021

DOI: https://doi.org/10.21203/rs.3.rs-1030889/v1

License: () (1) This work is licensed under a Creative Commons Attribution 4.0 International License. Read Full License 


\section{Abstract}

Aim: Antibiotic resistance is a public health concern that is linked to increased mortality, morbidity, extended hospital stays, decreased productivity, and therefore, increased financial implications. The source and dissemination of antimicrobial resistance have been linked to environmental factors, including dust storms. There is clear evidence that there has been a rapid increase in temperature in the past decades which increases the risk of sandstorms in places that had never previously experienced this phenomenon. The aim of this study is to isolate medically important micro-organisms in atmospheric air samples from outdoor spaces and inside hospital wards during sandstorms and to characterize their antimicrobial sensitivity profiles to common antibiotics.

Findings: Eighty-four colonies were isolated from the target sites and identified as Staphylococcus aureus, coagulase-negative Staphylococcus, Bacillus spp., Acinetobacterspp., from hospital air samples, and Pseudomonas spp., and Staphylococcus spp. from outdoor air samples. Multi-drug resistance patterns were observed for isolates obtained from both indoor and outdoor air samples. PCR showed $6.5 \%$ of $S$. aureus contained mecA.

Conclusion: This finding supports the notion that atmospheric air during dust storms can be counted as a source of antibiotic resistant bacteria which merits more attention especially with global warming and climate change contributing to extreme weather events.

\section{Introduction}

In the current clinical settings, antibiotic resistance and its effects are the greatest healthcare concerns. The latest body of evidence implicates the environment as a critical element in transmitting and evolving antibiotic-resistant bacteria [1]. However, clear evidence that directly links the evolutionary and ecological factors to the emergence of resistant genes in microbes is currently lacking. Therefore, such research gaps call for more lucid explanations of the development and evolution of resistant genes, their mobilisation, transfer, and dissemination in the environment.

Antimicrobial resistance is responsible for a large proportion of mortalities on an annual basis. Research has suggested a projection in its increase in the coming years, which has led the World Health Organization (WHO) to make a recognition regarding its threats as a public health hazard [2]. As far as the history of recognising danger and endeavours against antimicrobial resistance is concerned, activities aimed at controlling the development of resistance have primarily been initiated in the clinical settings and at the community level. Very recently, the problem has also commenced in the environmental and agricultural context [3]. The aim to solve this problem is the reduction of the transmission and prevention in selecting the resistant bacteria, whilst undertaking antibiotic therapies.

WHO has issued the warning that the modern world is fast heading to the post-antibiotic times, leading to uncontrollable morbidity and mortality [4]. Furthermore, the hospitals and healthcare facilities are fast turning into the hubs of extremely drug-resistant microorganisms [5], whereby even routine surgical procedures like cancer surgery and other surgical operations may become extremely risky. A scientific report forecasts that by the middle of the twenty-first century, under the current circumstances, if there is no improvement in the antibiotic-resistance development scenario, the population figures of the world shall be from 11 million to 440 million lesser than projected. Similarly, the world's economic losses will make the economy shrink by anywhere ranging from $0.06-$ $3.1 \%$ [6]. Antimicrobial resistance is normally linked to substantial mortality, morbidity, extended hospital stays, and higher financial implications.

Climate change and its effect on environment and microbiome are important issues of concern. The insufficiency of such a knowledge base regarding how, why, and when the environmental factors become contributory to the development of resistance make antimicrobial resistance risk reduction quite a difficult task. For example, unidentified microbes transported by desert dust storms across the Atlantic were shown to be the causative agents in coral diseases, contributing to the decline of reef ecosystems in the Caribbean basin [7]. The involvement of environmental factors as contributors to sourcing and dissemination of antimicrobial resistance in clinical settings has gained considerable recognition. The latest body of evidence implicates the environment as a critical element in transmitting and evolving antibiotic resistant bacteria [1]. For example, shunt infections in the operating theatres were caused by airborne bacteria rather than originating from patients' skin [8-9] and also contamination in operating theatres air conditioning systems was to blame for some postcataract infections in a hospital [10].

Kuwait is a country in the western part of the Asian continent, with vast deserts and an abundance of dust storms in hot and dry weather conditions. A greater part of the country consists of deserts and an abundance of dust storms in hot and dry weather conditions. A research study based on the desert of Kuwait found 147 colony-forming units (CFU) in the desert dust [11]. Global warming has affected this region with higher temperatures $\left(1.5^{0} \mathrm{C}\right.$ to $2^{0} \mathrm{C}$ increase in temperature annually), and increase in the frequency of rising dust and longer duration of sandstorms (https://www.ecomena.org sclimate-change-kuwait).

It also faces risks from sea level rise, lack of rainfall, and biodiversity degradation (https://thearabweekly.com/climate-change-endangers-quality-life-kuwait).

Our aim in this study was to identify if any antibiotic resistant bacteria that are significant in clinical settings were found in air during sandstorms.

\section{Materials And Methods}

\section{Sampling}

Air sampling was performed in two hospitals $(X$ and $Y)$ in Kuwait Air samples were collected from common areas of different departments; the Intensive Care Unit (ICU), Cardiac Care Unit (CCU), and Operating Theatres (OT) with consideration of beds, patients, and staff numbers in each department. The temperatures ranged between $18^{\circ} \mathrm{C}-20^{\circ} \mathrm{C}$. The ICU and $\mathrm{CICU}$ (cardiac ICU) had bed capacity of 40 and 4 respectively. Only one operating theatre was available for sample collection. Thirty-four airborne isolates of dust raised during sandstorms were collected from open areas of two different sites (Site 1 and Site 2 ). 
Sample collection was achieved by SKC Biostage ${ }^{\text {TM }}$ device onto R2A agar (Reasoner's 2A agar - a culture medium for the bacterial examination of drinking water) and nutrient agar (culture medium used for the growth of non-fastidious bacteria) containing $50 \mu \mathrm{g} / \mathrm{ml}$ cycloheximide, $10 \mu \mathrm{g} / \mathrm{ml} \mathrm{nystatin}$ and $10 \mu \mathrm{g} / \mathrm{ml}$ of one of these antibiotics: erythromycin, streptomycin, tetracycline and nalidixic acid.

Samples were collected in triplicates from each target site; thirty-four collected from an open air site during a sandstorm and thirty-nine from the two target hospitals from inpatient departments; operating theatres, intensive care units, and cardiac care units of the two hospitals equipped with high-efficiency particulate air (HEPA) filtration systems.

Single colonies from the collected samples were isolated and incubated for 3-4 days at $37^{\circ} \mathrm{C}$ to enhance growth. Upon visible growth, each isolate was put in an antibiotic plate and stored with glycerol supplement at $-80^{\circ} \mathrm{C}$ (Table 1$)$.

\section{Identification Of Airborne Bacteria}

Genomic DNA from single isolated colonies from each antibiotic plate were extracted using Wizard genomic DNA purification kit (promega). Amplifications of

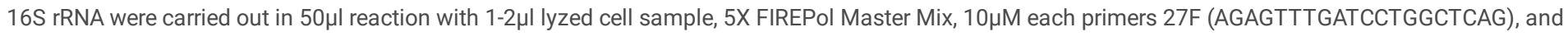
1492R (GGTTACCTTGTTACGACTT) under the following conditions: $5 \mathrm{~min}$ at $95^{\circ} \mathrm{C}$ followed by 35 cycles of $30 \mathrm{~s}$ at $94^{\circ} \mathrm{C}, 30 \mathrm{~s}$ at $54^{\circ} \mathrm{C}$, and 2 min at $72^{\circ} \mathrm{C}$, and 1 cycle of $10 \mathrm{~min}$ at $72^{\circ} \mathrm{C}$. The PCR products were separated on $1 \%$ agarose gel. Amplified DNA fragments were purified using Wizard SV Gel \& PCR purification kit (Promega). The PCR amplicons were then sequenced using ABI 3130xI genetic analyser. BioNumerics v.7.1 (Applied Maths, Ghent, Belgium) was used to analyse the sequences. Identification at the species level was performed by comparison with the Ribosomal Database Project database (http://rdp.cme.msu.edu/) and by using BLAST (http://blast.ncbi.nlm.nih.gov/Blast.cgi).

\section{Antibiotic-resistance Screening}

The susceptibility of the bacterial isolates to different antibiotics was determined in triplicate using the standard disk diffusion and E-test methods and analysed (CLSI ${ }^{24}$ and EUCAST).

\section{Detection of mecA gene}

PCR of mecA gene was carried out in 50 $\mu$ reaction with 1-2 $\mu$ l lyzed cell sample, 5X FIREPol Master Mix, 10 $\mu \mathrm{M}$ each primer forward 5'AGGCCCGGGAACGTATTCAC-3' and reverse $5^{\prime}$-GAGGAAGGTGGGGATGACGT-3' under the following conditions: an initial denaturation step at $94^{\circ} \mathrm{C}$ for 5 min, followed by 30 cycles of $94^{\circ} \mathrm{C}$ for $30 \mathrm{sec}, 52^{\circ} \mathrm{C}$ for $30 \mathrm{sec}$, and $72^{\circ} \mathrm{C}$ for $30 \mathrm{sec}$, with a final extension at $72^{\circ} \mathrm{C}$ for 5 min. The PCR products were separated on $1 \%$ agarose gel. Amplified DNA fragments were purified using Wizard SV Gel \& PCR purification kit (Promega). The sequences were analysed using BLAST (http://blast.ncbi.nlm.nih.gov)

\section{Results}

A total number of 84 colonies were isolated from 34 air sample agar plates. These isolates are shown is Table 1.

Table 1

The number of bacteria

identified in samples.

\begin{tabular}{|ll|}
\hline Sample & Number \\
\hline Staphylococci & 46 \\
Bacillus & 20 \\
\hline Acinetobacter & 5 \\
\hline Pseudomonas & 2 \\
Others & 11 \\
\hline Total & 84 \\
\hline
\end{tabular}

\section{Hospital Air Samples}

Staphylococci $(n=26)$

Twenty sixStaphylococci were recovered from hospital air samples Including 7 S. epidermidis, 6 S. capitis, 5 S. hominis, 2 S. haemolyticus and 2 S. warneri, 1 S. caprae, 1S. petrasii, 1 S. aureus, and 1 S. lugdunensis.

The graph in (Figure.1) shows the overall resistance patterns of Staphylococcus species obtained and cultured from air against medically important antibiotics used in clinical settings. Majority (73\%, 19 out of 26) of the Staphylococcus colonies were resistant to trimethoprim-sulfamethoxazole followed by $50 \%$ resistant to erythromycin, and $23 \%$ resistant to ciprofloxacin. $19 \%$ of the Staphylococci isolates were resistant to cefoxitin, whilst $15 \%$ were resistant to tetracycline and clindamycin. A smaller number were resistant to oxacillin (12\%), kanamycin (12\%), linezolid (8\%), streptomycin and vancomycin (4\% each).

Page $3 / 16$ 
Notably, all S. epidermidis isolates were found to be resistant to trimethoprim-, sulfamethoxazole whilst most (4 out of 7 isolates) were resistant to at least three different classes of antibiotics, including trimethoprim-sulfamethoxazole, erythromycin, tetracycline, kanamycin, clindamycin, and ciprofloxacin. Nevertheless, only one isolate showed resistance to oxacillin.

S. capitis isolates were resistant to trimethoprim-sulfamethoxazole and erythromycin but sensitive to oxacillin.

$57 \%(n=5)$ of the $S$. hominis isolates also showed resistance to trimethoprim-sulfamethoxazole. Two of these were found to show multiple drug resistance pattern, including oxacillin.

The two S. haemolyticus isolates showed cross-resistance to trimethoprim-sulfamethoxazole and erythromycin and were also found to be resistant to another class of antibiotic (either ciprofloxacin or tetracycline), but not to oxacillin. The rest of the Staphylococci isolates showed resistance to only one or two antibiotic classes (Table 2).

Table 2

Resistance patterns of Staphylococcus numbers isolated from hospital air samples.

\begin{tabular}{|ll|}
\hline Antibiotic & Numbers \\
\hline Amikacin (AK) & 0 \\
\hline Erythromycin (EM) & 13 \\
\hline Cefoxitin (FX) & 5 \\
\hline Gentamicin256 (GM256) & 0 \\
\hline Gentamicin1024 (GM1024) & 0 \\
\hline Linezolid (LZ) & 2 \\
\hline Oxacillin (OX) & 3 \\
\hline Trimethoprim-Sulfamethoxazole (SXT) & 0 \\
\hline Streptomycin (STP/SM) & 1 \\
\hline Tetracycline (TET/TC) & 4 \\
\hline Trimethoprim (TR) & 19 \\
\hline Vancomycin (VA) & 1 \\
\hline Kanamycin (KM) & 3 \\
\hline Clindamycin (CM) & 4 \\
\hline Ciprofloxacin (CI) & 6 \\
\hline
\end{tabular}

Bacillus $(\mathbf{n}=\mathbf{8})$

Eight isolates of Bacillus were obtained and cultured from hospital air samples (Table3), consisting of 5 B. subtilis, 1 B. cereus, 1 B. clausii, and 1 B. haynassu.

$63 \%(n=)$ ) of the Bacillus isolates were found to be resistant to oxacillin, 38\% $(n=)$ were resistant to erythromycin, and another $38 \%(n=)$ were resistant to trimethoprim-sulfamethoxazole. $25 \%(n=)$ were resistant to mupirocin, whilst the following antibiotics were found to have one $(13 \%)$ isolate to which it was resistant to:cefoxitin, streptomycin, tetracycline, vancomycin, kanamycin, and clindamycin (Figure.2). Analysis for the resistance pattern (based on MIC per species indicates three out of five isolates of $B$. subtilis showed resistance to oxacillin. Two of these isolates showed multiple drug resistance patterns. $B$. cereus isolate was resistant to oxacillin and trimethoprim, whilst $B$. haynassu was resistant to mupirocin and streptomycin. $B$. clausii isolate showed multiple drug resistance, including erythromycin, cefoxitin, oxacillin, vancomycin, kanamycin, and clindamycin (Table 3). 
Table 3

Resistance pattern of Bacillus numbers

isolated from hospital air samples.

\begin{tabular}{|ll|}
\hline Antibiotic & Numbers \\
\hline Amikacin (AK) & 0 \\
\hline Erythromycin (EM) & 3 \\
\hline Cefoxitin (FX) & 1 \\
\hline Gentamicin256 (GM256) & 0 \\
\hline Gentamicin1024 (GM1024) & 0 \\
\hline Mupirocin (MU) & 2 \\
\hline Oxacillin (OX) & 5 \\
\hline Streptomycin (STP/SM) & 1 \\
\hline Tetracycline (TET/TC) & 1 \\
\hline Trimethoprim (TR) & 3 \\
\hline Vancomycin (VA) & 1 \\
\hline Kanamycin (KM) & 1 \\
\hline Clindamycin (CM) & 1 \\
\hline
\end{tabular}

Acinetobacter $(n=5)$

There were five isolates of Acinetobacter cultured from hospital air, four of which were $A$. baumanii, whilst the other one was $A$. Iwoffii . 80\%(n=) of $A$. baumanii were resistant to imipinem, meropenem, and tetracycline while $40 \%(n=1)$ were resistant to amikacin (Figure.3). This also implies multiple drug resistance patterns in A. baumanii. A. Iwoffii did not show resistance to any of the tested antibiotics (Table 4).

Table 4

Resistance pattern of Acinetobacter numbers isolated from hospital air samples.

\begin{tabular}{|ll|}
\hline Antibiotic & Numbers \\
\hline Amikacin (AK) & 2 \\
\hline Imipenem (IP) & 4 \\
\hline Meropenem (MP) & 4 \\
\hline Tetracycline (TET/TC) & 4 \\
\hline
\end{tabular}

\section{Other Isolates}

There was a total of ten other isolates obtained from hospital air samples. These consisted of Micrococcus aloeverae, Micrococcus luteus, Corynebacterium simulans, Luteimonas terrae, Agrobacterium salinitolerans, Corynebacterium amycolatum, Corynebacterium casei, and Escherichia fergusonii. All of these isolates, except for L. terrae, C. casei, and E. fergusonii, showed resistance to Mupirocin. Moreover, multiple drug resistance was exhibited by $A$. salinitolerans, M. aloeverae, and C. amycolatum.

\section{Outdoor Isolates}

\section{Bacillus ( $\mathrm{N}=12)$}

Ten out of the twelve isolates across all the species showed resistance to at least three different classes of antibiotics, including Oxacillin. Based on MIC values, three showed resistance to imipenem, vancomycin, and linezolid and only one showed resistance to meropenem, amikacin, and ciprofloxacin, and none showed resistance to colistin and gentamicin (Table 5).

Both Solibacillus isronensis B3W22 isolates showed resistance to erythromycin, cefoxitin, imipenem, mupirocin, oxacillin, streptomycin, tetracycline, and trimethoprim (Figure.4). 
Table 5

Resistance pattern of Bacillus numbers isolated from outdoor air samples.

\begin{tabular}{|ll|}
\hline Antibiotic & Numbers \\
\hline Amikacin (AK) & 1 \\
\hline Ciprofloxacin (CI) & 1 \\
\hline Colistin (CT) & 0 \\
\hline Erythromycin (EM) & 6 \\
\hline Cefoxitin (FX) & 5 \\
\hline Gentamicin256 (GM256) & 0 \\
\hline Imipenem (IP) & 3 \\
\hline Linezolid (LZ) & 3 \\
\hline Meropenem (MP) & 1 \\
\hline Mupirocin (MU) & 7 \\
\hline Oxacillin (OX) & 10 \\
\hline Streptomycin (SM) & 5 \\
\hline Ticarcillin (TC) & 3 \\
\hline Teicoplanin (TP) & 1 \\
\hline Trimethoprim (TR) & 7 \\
\hline Vancomycin (VA) & 3 \\
\hline
\end{tabular}

Pseudomonas $(\mathrm{N}=2)$

Two isolates of $P$ seudomonas spp. were obtained consisting of $P$. xanthomarina and $P$. xiamenensis DSM22326. Both showed resistance to Imipenem. $P$. xiamenensis exhibited multiple drug resistance pattern, including resistance to ciprofloxacin, linezolid and imipenem (Table 6) (Figure 5).

Table 6

Resistance pattern of Pseudomonas numbers isolated from outdoor air samples.

\begin{tabular}{|ll|}
\hline Antibiotic & Numbers \\
\hline Amikacin (AK) & 0 \\
\hline Ciprofloxacin (CI) & 1 \\
\hline Erythromycin (EM) & 0 \\
\hline Cefoxitin (FX) & 0 \\
Imipenem (IP) & 2 \\
\hline Linezolid (LZ) & 1 \\
\hline
\end{tabular}

Staphylococcus $(\mathbf{N}=\mathbf{2 0})$

A total of 20 isolates were obtained from outdoor air samples. These consisted of the following: three $S$. arlettae, three S. epidermidis, three S. hominis and three S. xylosus, two S. cohnii and three S. haemolyticus, and one S. aureus, 1 S. equorum, 1 S. piscifermentans, and 1 S. saprophyticus.

$95 \%$ (19 out of 20 isolates) showed resistance to oxacillin, $65 \%$ (13 out of 20 ) to trimethoprim and $60 \%$ (12 out of 20 ) were resistant to erythromycin. $40 \%$ ( 8 out of 20 isolates) showed resistance to teicoplanin, and 35\% (7 out of 20) showed resistance to cefoxitin and mupirocin. There was 20\% (4 out of 20) resistance to clindamycin as well as imipenem, whilst there was $15 \%$ (3 out of 20) resistance to tobramycin. Additionally, there were $10 \%$ ( 2 out of 20 ) resistance to each of chloramphenicol, kanamycin, sulfamethoxazole-trimethoprim, and $5 \%$ (1 out of 20) resistance to each of amikacin and ciprofloxacin (Figure 6).

All the S. arlettae isolates exhibited cross-resistance to oxacillin and trimethoprim, and all three showed multidrug resistance patterns. However, none was resistant to vancomycin, imipenem, ciprofloxacin, and linezolid.

For S. epidermidis, S. hominis, and S. xylosus isolates, aside from all being resistant to oxacillin, only one isolate from each species showed resistance to less than three drug classes. All S. haemolyticus isolates also showed multiple drug resistance patterns. In fact, only 5 out of the 20 isolates showed resistance to only two classes of antibiotics. Nevertheless, none of these isolates exhibited resistance to gentamicin, linezolid, teicoplanin, and vancomycin (Table 7). 
Table 7

Resistance pattern of Staphylococcus numbers

isolated from outdoor air samples.

\begin{tabular}{|ll|}
\hline Antibiotic & Numbers \\
\hline Amikacin (AK) & 1 \\
\hline Ciprofloxacin (Cl) & 1 \\
\hline Chloramphenicol (CL) & 2 \\
\hline Clindamycin (CM) & 4 \\
\hline Erythromycin (EM) & 12 \\
\hline Cefoxitin (FX) & 7 \\
\hline Gentamicin256 (GM256) & 0 \\
\hline Gentamicin1024 (GM1024) & 0 \\
\hline Imipenem (IP) & 4 \\
\hline Kanamycin (KM) & 2 \\
\hline Linezolid (LZ) & 0 \\
\hline Meropenem (MP) & 7 \\
\hline Oxacillin (OX) & 19 \\
\hline Trimethoprim-Sulfamethoxazole (SXT) & 2 \\
\hline Tobramycin (TM) & 3 \\
\hline Ticarcillin (TC) & 8 \\
\hline Teicoplanin (TP) & 0 \\
\hline Trimethoprim (TR) & 13 \\
\hline Vancomycin (VA) & 0 \\
\hline
\end{tabular}

\section{Detection and Amplification of mecA Gene PCR}

All S. aureus isolates were screened for the presence of mecA gene by PCR. mecA gene was detected in only 3 S. aureus - (6.5\%) isolated from ICU.

\section{Discussion}

Kuwait, a country in the Gulf area of the Middle East (southwest Asia), is mostly a dry desert with regular sandstorms. Occasionally, sandstorms could result in closures of operating theatres due to high levels of dust in the atmospheric air. Air samples from clinical and outdoor settings often share properties and similar distribution of bacteria [7], therefore, it is important to study the impact of the air during sandstorms and rising dust on the indoor hospital air. Increasingly high temperatures and hot climates, coupled with high population and over prescription of antibiotic medication, may play a significant role in the presence of

The sand and dust effectively impact human health, the environment, and the economy of countries. Its damage to the infrastructures and interruption to transportation is evident. But the long-term effect of these particles on human health explored here should be studied more [12]. The airborne dust and its particle size determine the amount of impact on people's health. World scientists have linked environmental conditions like dust storms to the increasing pattern of bacterial infections amongst the populations. When the dust particles are inhaled in hot dry weather, the nose and throat mucosa are damaged, giving rise to bacterial infections. This extreme event is prevalent in many parts of the world because of its ability to travel through the earth's atmosphere. However, the main sources of dust are the arid regions of North Africa, the Arabian Peninsula, China, and Central Asia [12]. The Middle East, especially the places like Kuwait and Dubai, experience dusty weather more commonly when compared to others. Kuwait has a subtropical desert climate that results in extremely hot and dry summers with a very short winter. The oil industries present here contribute to toluene and sulphur dioxide pollution. The increase in dust storms every year certainly plays an important role in the antibiotic resistance amongst the people of Kuwait, and it should lead to more studies.

In this study, we were able to isolate the following bacteria from hospital air samples: Staphylococcus aureus, coagulase-negative Staphylococcus, Bacillus spp., Acinetobacter spp., Micrococcus spp., Corynebacterium simulans, Luteimonas terrae, Agrobacterium salinitolerans, Corynebacterium amycolatum, Corynebacterium casei, and Escherichia fergusonii. Our findings are similar to the study conducted by Toar et al. [13] who studied hospital operating room air samples and found the following isolates: Klebsiella pneumoniae, coagulase-negative Staphylococcus, $*$ [1] and Bacillus subtilis. In another study Solomon et al. [14] collected hospital indoor air samples via passive air sampling method. They found coagulase-negative Staphylococci (29.6\%), Staphylococcus aureus (26.3\%), Pseudomonas aeruginosa (5.3\%), Acinetobacter spp., (9.5\%), Enterococci species, Enterococcus faecalis and Enterococcus faecium (16.5\%), Acinetobacter species (9.5\%), and Escherichia coli (5.8\%). Similar types of bacteria were found in our study, however, only they did not isolate Bacillus spp., and we did not isolate Enterococcus spp. In another study by Kunwar et al.[15] across eight hospitals in Kathmandu, Nepal, isolated bacteria from hospital air 
samples included Staphylococcus aureus (47.18\%), Pseudomonas spp. (1.82\%), and others such as coagulase negative Staphylococcus, Streptococcus spp., Micrococcus spp., Bacillus spp., E. coli, and Proteus spp. Like our study, Kunwar et al. were able to isolate Staphylococcus spp. and Bacillus spp., but not Acinetobacter.

In terms of Staphylococcus, the isolates in our study exhibited resistance to trimethoprim, erythromycin, ciprofloxacin, cefoxitin, tetracycline, and clindamycin; only $12 \%$ were resistant to oxacillin. In the study performed by Solomon et al. ${ }^{14}$, methicillin resistance was observed in $38.9 \%$ of the isolates, higher than this study. In other studies performed by Toar et al. [13] and Kunwar et al. [15], the results of antibiotic sensitivity testing for their isolates were not reported. Solomon et al. [14] found that Acinetobacter were resistant to gentamicin, trimethoprim-sulfamethoxazole, and ciprofloxacin; whereas, in our study, we found that Acinetobacter were resistant to imipenem, meropenem, and tetracycline. Our finding of multidrug resistance pattern for Acinetobacter was different from Solomon et al. In another study conducted by Shamsizadeh et al. [16], Acinetobacter resistant to ceftazidime, imipenem, and gentamicin were isolated from the intensive care units.

\section{Outdoor Air Isolates}

We isolated Pseudomonas, Staphylococcus aureus, and coagulase-negative Staphylococcus, from outdoor air samples which were similar to the findings from studies as discussed below. In one study [17], the dominant species isolated from a school in Nigeria were Escherichia coli, Micrococcus spp., Klebsiella spp., Pseudomonas spp., and Staphylococcus spp. In another study [18] indoor and outdoor air samples in a school in India were tested and Micrococcus, Staphylococcus, Streptococcus, Bacillus, Legionella, Pseudomonas, Klebsiella, and Mycobacteria were isolated from both samples. They observed differences between locational indoor concentrations of the microorganisms depending on which areas were more frequently visited and showed environmental outdoor microorganisms can spread indoors.

Li et al. [19] performed a global survey of antibiotic-resistance genes from urban air and found that there were 30 antibiotic-resistance gene subtypes resistant to the following classes of antibiotics: beta-lactam, quinolones, tetracyclines, macrolides, aminoglycosides, sulphonamides, and vancomycin. Cities that were included in the study were Haikou, Hong Kong, Guangzhou, Shanghai, Beijing in China, Bandung in Indonesia, San Francisco in the USA, Melbourne and Brisbane in Australia, Singapore, Paris and Tours in France, amongst others. This study highlighted the notion that urban air across the globe can contain antibiotic-resistant microorganisms.

In this study, various species of Staphylococcus and Bacillus with different antimicrobial resistance profiles were found from both outdoor and hospital air samples during sandstorms. Comparing the resistance patterns of the isolates obtained from outdoor air samples with hospital indoor air samples, shows remarkably more Staphylococcus isolates obtained from atmospheric outdoor air samples were resistant to oxacillin ( $95 \%$ outdoor vs $12 \%$ hospital). Resistance to trimethoprim and erythromycin amongst outdoor and hospital Staphylococcus isolates were: $65 \%$ outdoor vs $73 \%$ hospital and $60 \%$ outdoor and $50 \%$ hospital, respectively. In terms of multiple drug resistance patterns, $75 \%$ of the outdoor isolates versus $42 \%$ of hospital isolates were resistant to at least three different classes of antibiotics. However, outdoor isolates did not exhibit resistance to linezolid and vancomycin, whilst one isolate collected from hospital air, was resistant to vancomycin and two isolates were resistant to linezolid. This could translate to a higher occurrence of methicillin-resistant Staphylococcus spp. and multidrug-resistant strains in atmospheric outdoor air, nevertheless, the findings support the ubiquity of Staphylococcus spp. both in outdoor air and in hospital premises.

In contrast to the results of our study, Tamberkar et al., 2007 [20], showed that Staphylococcus were isolated more from the outdoor air samples than from indoor samples. The authors attributed this to shedders as being the sources of the high burden of Staphylococcus, which disperse large numbers of Grampositive cocci into the environment. In the same study, they were also able to isolate Pseudomonas aeruginosa, which had a higher concentration in the indoor air than outdoor air. In our present study, we were only able to isolate Pseudomonas spp. from hospital air samples.

Oxacillin resistance was detected in Bacillus isolates from both outdoor (83\%) and hospital (63\%) air isolates. There were also higher rates of resistance amongst outdoor air isolates against mupirocin ( $58 \%$ outdoor vs $25 \%$ hospital), trimethoprim ( $58 \%$ outdoor vs $38 \%$ hospital), and erythromycin ( $50 \%$ outdoor vs $38 \%$ hospital). Of the outdoor isolates, $75 \%$ showed resistance to more than three antibiotic classes, whilst amongst the hospital isolates, only $38 \%$ showed multiple drug resistance. Moreover, three of the outdoor isolates exhibiting multiple drug resistance patterns also were resistant to one or two of the following antibiotics: imipenem, linezolid, and vancomycin. Such findings support the notion of environmental Bacillus spp. (usually in soil) as a source of hospitalacquired infection [21] that would be harder to treat with available antibiotics.

From indoor air samples we identified Staphylococcus Species, Bacillus, Acinetobacter, and micrococcus. Outdoor air samples contained Bacillus, Pseudomonas, and Staphylococcus. In comparison, Bacillus and Staphylococcus were found from both outdoor and hospital air samples. Acinetobacter, Micrococcus, and Bacillus were not found in outdoor samples and indicated the possibility of contamination from outdoor environmental sources.

There was no isolated Acinetobacter from outdoor air samples. Acinetobacter survives best in water and soil, whereas our research was focused on collection of air samples. Acinetobacter can be found on human skin and can survive for long under unconducive settings, thus attributing possible contamination of indoor environment [16].

There are some limitations to our study, the sample collection method included both targeted and non-targeted bacteria. Different growth conditions and rates could affect the distribution of the bacteria in the samples. Overgrowing bacteria could inhibit the growth of the slow-growing bacteria through competition for resources and thus affect the results. The study only used data from two hospitals and two outdoor target sites, making statistical relationships for all hospitals in Kuwait difficult, moreover this is the first study in Kuwait to sample air during sand storms and therefore lack of prior research data limited the scope of the study analysis. 
In conclusion, antibiotic-resistance is a public health concern that greatly affects the progression and management of infective diseases. In this study, we have determined the types and the distribution of antibiotics-resistant bacteria present in the Kuwaiti air samples and compared the results with the bacteria present in the clinical settings. Dust rising from sandstorms contribute to the species of bacteria seen in clinical settings in Kuwait. Moreover, some bacteria tend to be able to survive for longer periods and thrive in the clinical settings, such as Acinetobacter that has the capability of remaining for prolonged periods in the environment. The exact nature and the extend of how environment plays its contributory role in propagation of antimicrobial resistance is still not clear. More attention should be paid to the environment as a critical contributing factor to link the ecological influences to the propagation of resistant bacteria in air.

*1 Coagulase-negative Staphylococcus include S. saprophyticus, S. epidermidis, S. hominis

\section{List Of Abbreviation}

\begin{tabular}{|ll|}
\hline CCU & Cardiac Care Unit \\
\hline CFU & Colony-Forming Units \\
CICU & Cardiac Intensive Care Unit \\
\hline DNA & Deoxyribonucleic Acid \\
\hline HEPA & High-Efficiency Particulate Air \\
ICU & Intensive Care Unit \\
\hline MIC & Minimum Inhibitory Concentration \\
\hline PCR & Polymerase Chain Reaction \\
\hline R2A & Reasoner's 2A Agar \\
\hline RNA & Ribonucleic Acid \\
\hline Spp & Species, \\
\hline WHO & World Health Organization \\
\hline
\end{tabular}

\section{Declarations}

\section{Availability of data and materials}

All data generated or analysed during this study are included in this published article

\section{Conflicts of Interest}

No potential conflict of interest was reported by the authors.

\section{Funding Statement}

This work was supported by Kuwait University Research Administration Grant number RN01/15.

\section{Author contribution}

Sara Shamsah: Conceptualization, Methodology, Validation, Analysis, Investigation, Data Curation, and Writing,

Leila Vali: Methodology, Validation Analysis, Investigation, Data Curation, Writing - Review and Editing, Review, Supervision, and Editing.

Dana Al-Kayyalli: Analysis, Data Curation, Review and Editing.

Ali.A. Dashti: Review and Editing, Project Administration

\section{Acknowledgements}

I would like to acknowledge the Research Unit for Genomics, Proteomics and Cellomics Studies (OMICS) of the Health Sciences Centre, Kuwait University (Project No. SRUL02/13) for assisting in DNA sequencing

\section{References}

1. A. Singer, H. Shaw, V. Rhodes, and A. Hart, "Review of Antimicrobial Resistance in the Environment and Its Relevance to Environmental Regulators," Frontiers in Microbiology, vol. 7, pp. 1728-1728, 2016.

2. F. Prestinaci, P. Pezzotti., and A. Pantosti, “Antimicrobial resistance: a global multifaceted phenomenon", Pathogens and Global Health, vol. 109, no.7, pp. 309-318, 2015. 
3. B. Aslam, W. Wang, M. Arshad, M. Khurshid, S. Muzammil, M. Rasool, M. Nisar, R. Alvi, M. Aslam, M. Qamar, M. Salamat, and Z. Baloch "Antibioticresistance: a rundown of a global crisis", Infection and Drug Resistance, vol 11, pp. 1645-1658, 2018.

4. C. L. Ventola, "The antibiotic-resistance crisis: part 1: causes and threats", $P$ \& T: a peer-reviewed journal for formulary management, vol. 40, no. 4, pp. 277-283, 2015.

5. S. Zaman, M. Hussain, R. Nye, V. Mehta, K. Mamun, and N. Hossain, "A Review on Antibiotic-resistance: Alarm Bells are Ringing”. Cureus, vol. 9, p. e1403, 2017.

6. J. Taylor, M. Hafner, E. Yerushalmi, R. Smith, J. Bellasio et al., "Estimating the economic costs of antimicrobial resistance: Model and Results. Santa Monica”, CA: RAND Corporation, 2014. http://www.rand.org/pubs/research_reports/RR911.

7. N. Fierer, Z. Liu, M. Rodriguez-Hernandez, R. Knight, M. Henn, and M. T. Hernandez, "Short-term temporal variability in airborne bacterial and fungal populations," Applied and Environmental Microbiology, vol. 74, no1, pp. 200-207, 2008.

8. A. Duhaim, K. Bonner, K. McGowan, L. Schut, L. Sutton, and S. Plotkin, "Distribution of bacteria in the operating room environment and its relation to ventricular shunt infections: a prospective study" Child's Nervous System, vol. 7, no. 4, 1991.

9. Healio.com. "Troops returning from war at risk for infectious diseases". [online] Available at: https://www.healio.com/infectious-disease/emergingdiseases/news/print/infectious-disease-news/\%7B7c1734e7-56ad-4d31-b85e-289c96a480fa\%7D/troops-returning-from-war-at-risk-for-infectiousdiseases [Accessed 8 May 2019].

10. A. Pinna, D. Usai, L. Sechi, S. Zanetti, N. Jesudasan, P. Thomas, and J. Kaliamurthy, "An Outbreak of Post-Cataract Surgery Endophthalmitis Caused by Pseudomonas aeruginosa", Ophthalmology, vol. 116, no 12, pp. 2321-2326.e4, 2009.

11. D. Griffin, W., "Atmospheric movement of microorganisms in clouds of desert dust and implications for human health," Clinical Microbiology Reviews, vol. 20, no.3, pp. 459-477, 2007 [online] Available at 10.1128/CMR.00039-06. Accessed 28 January 2020.

12. Akthar S., Zahedi K., Bonapace T. Sand and Dust Storms in Asia and the Pacific: Opportunities for Regional Cooperation and Action. Bangkok: United Nations Publication; 2018.

13. M. M. Toar, E. Ardiansyah, M. R. Tala, S. M. Lumbanraja, H. S. Siregar, and D. Edianto, "Pattern and sensitivity test against bacteria in the air at Ob/Gyn operating room of central surgery installation in Central General Hospital (Rsup) Haji Adam Malik Medan," IOSR Journal of Nursing Health Science, vol. 7 no.1 pp. 28-33, 2007 [online] Available at http://www.iosrjournals.org/iosr-jnhs/papers/vol7-issue1/Version-10/E0701102833.pdf. Accessed 28 January 2020.

14. F. B. Solomon, F. W. Wadilo, A. A. Arota, and Y. L. Abrahan, "Antibiotic-resistant airborne bacteria and their multidrug resistance pattern at University teaching referral Hospital in South Ethiopia," Ann Clin Microbiol Antimicrob, vol. 16, no. 1, p. 29, 2017.

15. A. Kunwar, S. Tamrakar, S. Poudel, S. Sharma, and P. Parajuli, "Bacteriological assesssment of the indoor air of different hospitals of Kathmandu District," International Journal of Microbiology, 2019.

16. Z. Shamsizadeh, M. Nikaeen, B. Nasr Esfahani, S. H. Mirhoseini, M. Hatamzadeh, and A. Hassanzadeh, "Detection of antibiotic-resistant Acinetobacter baumannii in various hospital environments: potential sources for transmission of Acinetobacter infections," Environmental health and preventive medicine, vol, 22, no. 1, p. 44, 2017.

17. O. E. Udu-lbiam, A. V. Maduka, S. Chukwu Okoro, O. O. Olaosebikan, J. O. Orji, and E. C. Ekeghalu, "Microbiological analysis of outdoor air quality of male and female hostels in Ebonyi State University, Abakaliki, Ebonyi State, Nigeria," IOSR Journal of Pharmacy and Biological Sciences, vol. 11, no. 3, pp. 6873,2016

18. K. Bomala, G. Saramanda, T. Byragi Reddy, and J. Kaparapu, "Microbiological indoor and outdoor air quality Visakhapatnam City, India," International Journal of Current Research, vol. 8 no. 4, pp. 29059-29062, 2016, [online] Available at https://www.researchgate.net/publication/301693064. Accessed 28 January 2020.

19. J. Li, J. Cau, Y. Zhu, Q. Chen, F. Shen, Y. Wu, S. Xu, H. Fan, G. Da, R. Huang, J. Wang, A. L. de Jesus, L. Morawska, C. K. Chan, J. Peccia, and M. Yao, “Global survey of antibiotic-resistance genes in urban air," Environmental Science \& Technology, vol. 52, no. 19, pp. 10975-10984, 2018.

20. G. H. Tamberkar, P. B. Gulhane, and B. B. Bhokare, "Studies on environmental monitoring of microbial air flora in the hospitals," Journal of Medical Sciences, vol. 7, no. 1, pp. 67-73, 2007.

21. Clinical and Laboratory Standards Institute Performance standards for antimicrobial susceptibility testing. 29th ed. CLSI Supplement M100 [online]. Available at: "http://em100.edaptivedocs.net/GetDoc.aspx? doc=CLSI\%20M100\%20ED29:2019\&sbssok=CLSI\%20M100\%20ED29:2019\%20TABLE\%202C\&format=HTML" \I “CLSI\%20M100\%20ED29:2019\%20TABLE\%202C" http://em100.edaptivedocs.net/GetDoc.aspx? doc=CLSI\%20M100\%20ED29:2019\&sbssok=CLSI\%20M100\%20ED29:2019\%20TABLE\%202C\&format=HTML\#CLSI\%20M100\%20ED29:2019\%20TABLE\%2 (Accessed: 23 October 2019).

\section{Figures}


20

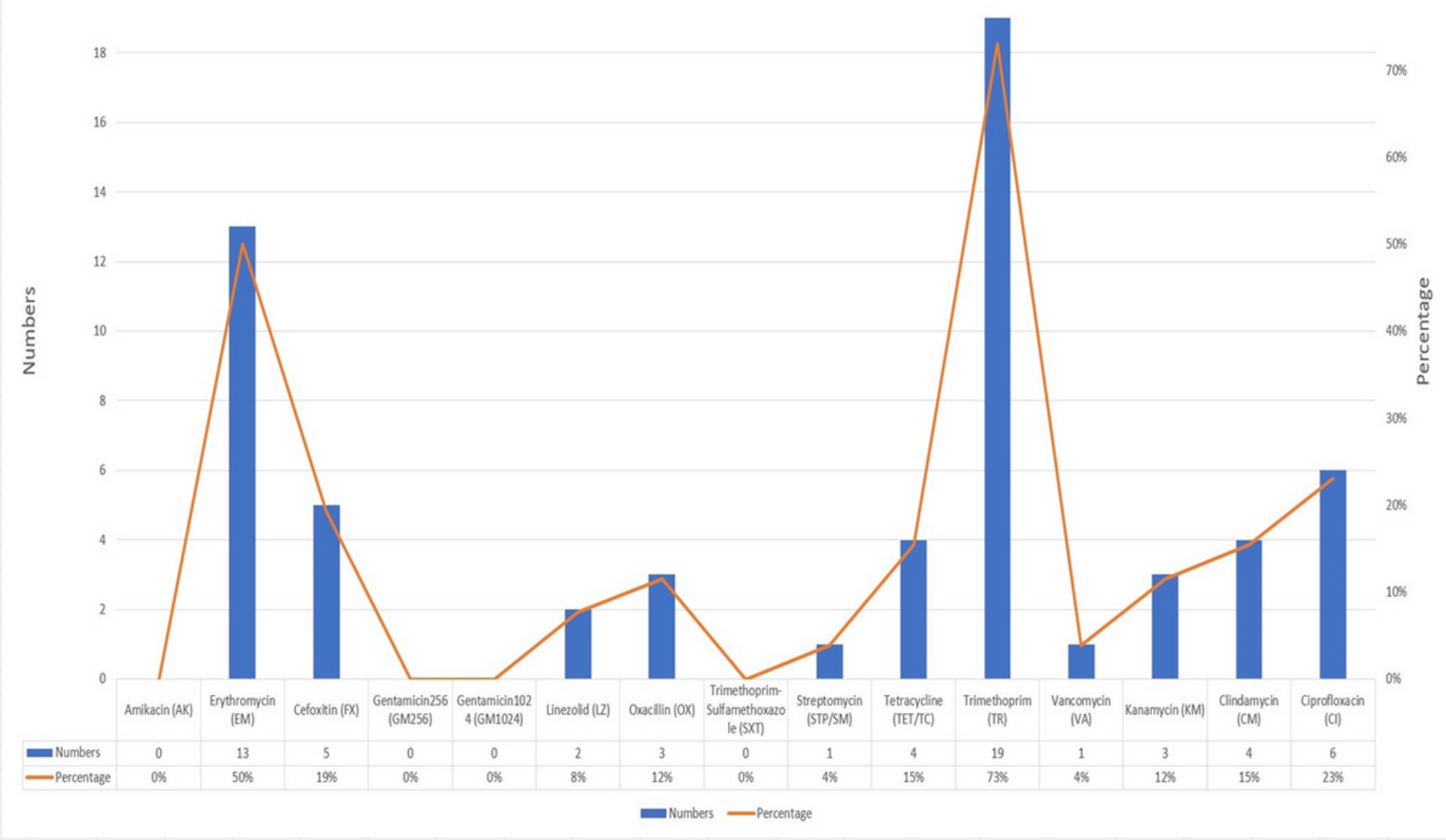

Figure 1

The graph in (Figure.1) shows the overall resistance patterns of Staphylococcus species obtained and cultured from air against medically important antibiotics used in clinical settings. 


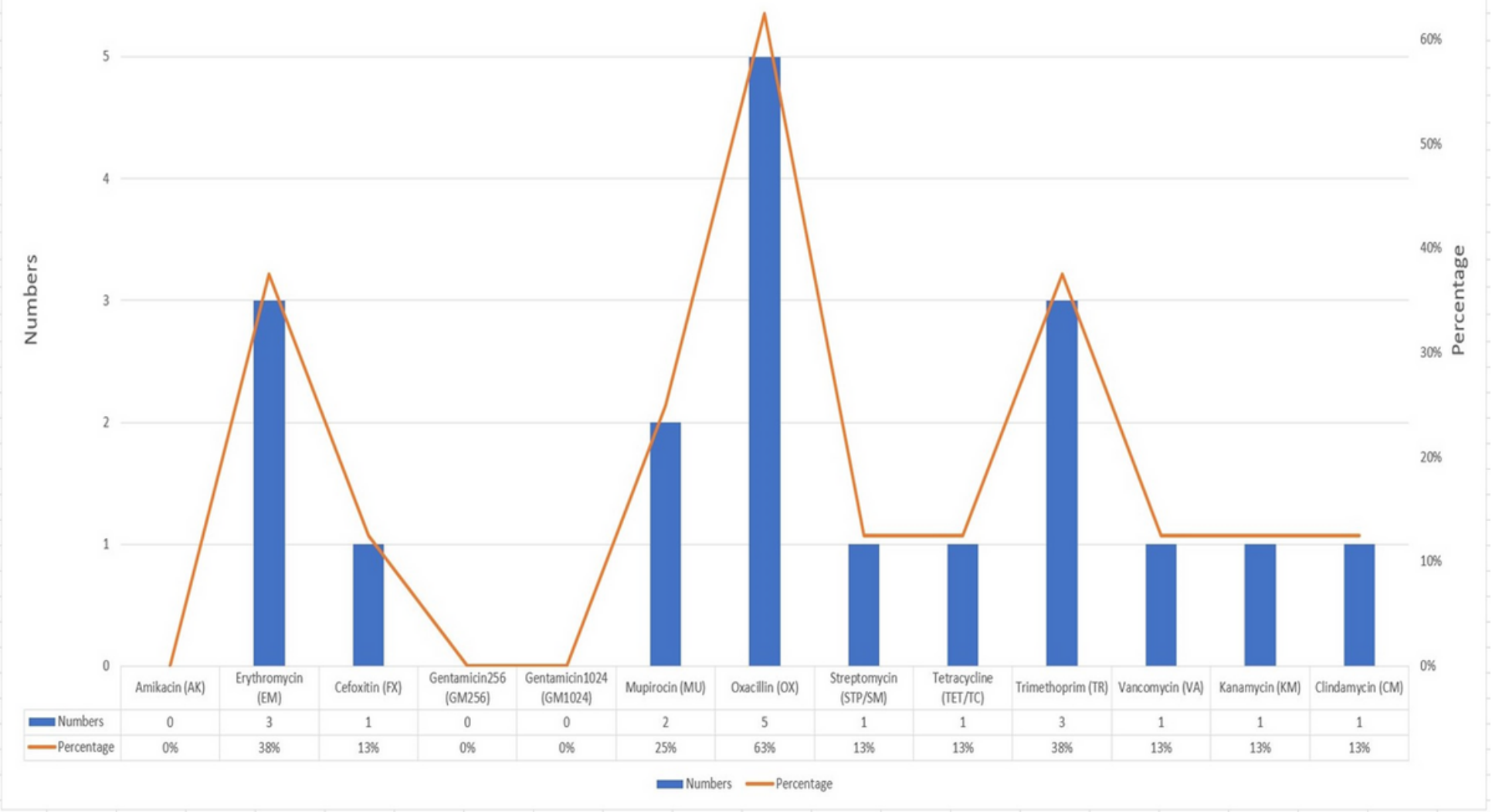

\section{Figure 2}

$25 \%(n=)$ were resistant to mupirocin, whilst the following antibiotics were found to have one $(13 \%)$ isolate to which it was resistant to:cefoxitin, streptomycin, tetracycline, vancomycin, kanamycin, and clindamycin (Figure.2). 


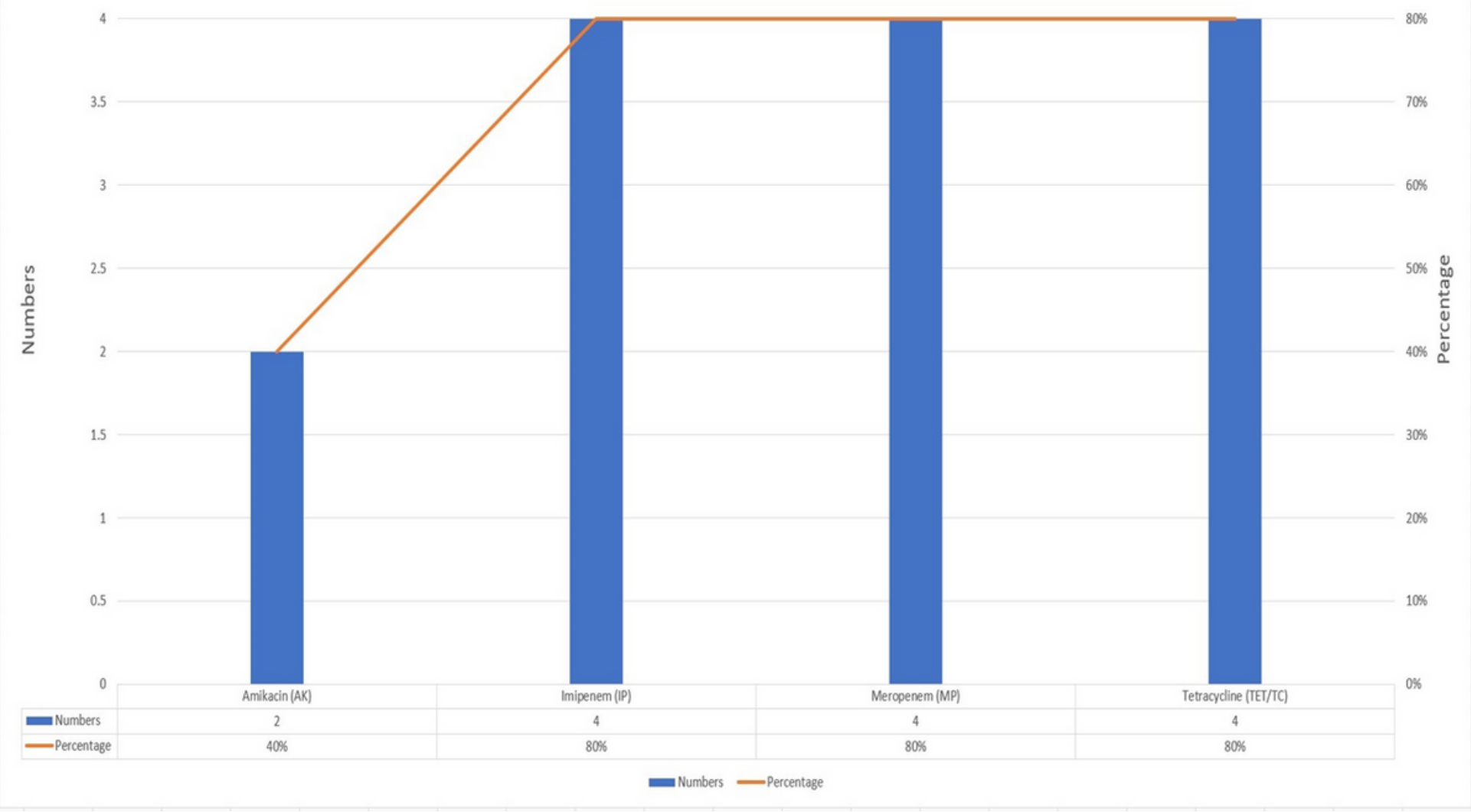

\section{Figure 3}

There were five isolates of Acinetobacter cultured from hospital air, four of which were A. baumanii, whilst the other one was A. Iwoffii . $80 \%(n=)$ of $A$. baumanii were resistant to imipinem, meropenem, and tetracycline while $40 \%(n=1)$ were resistant to amikacin (Figure.3). 


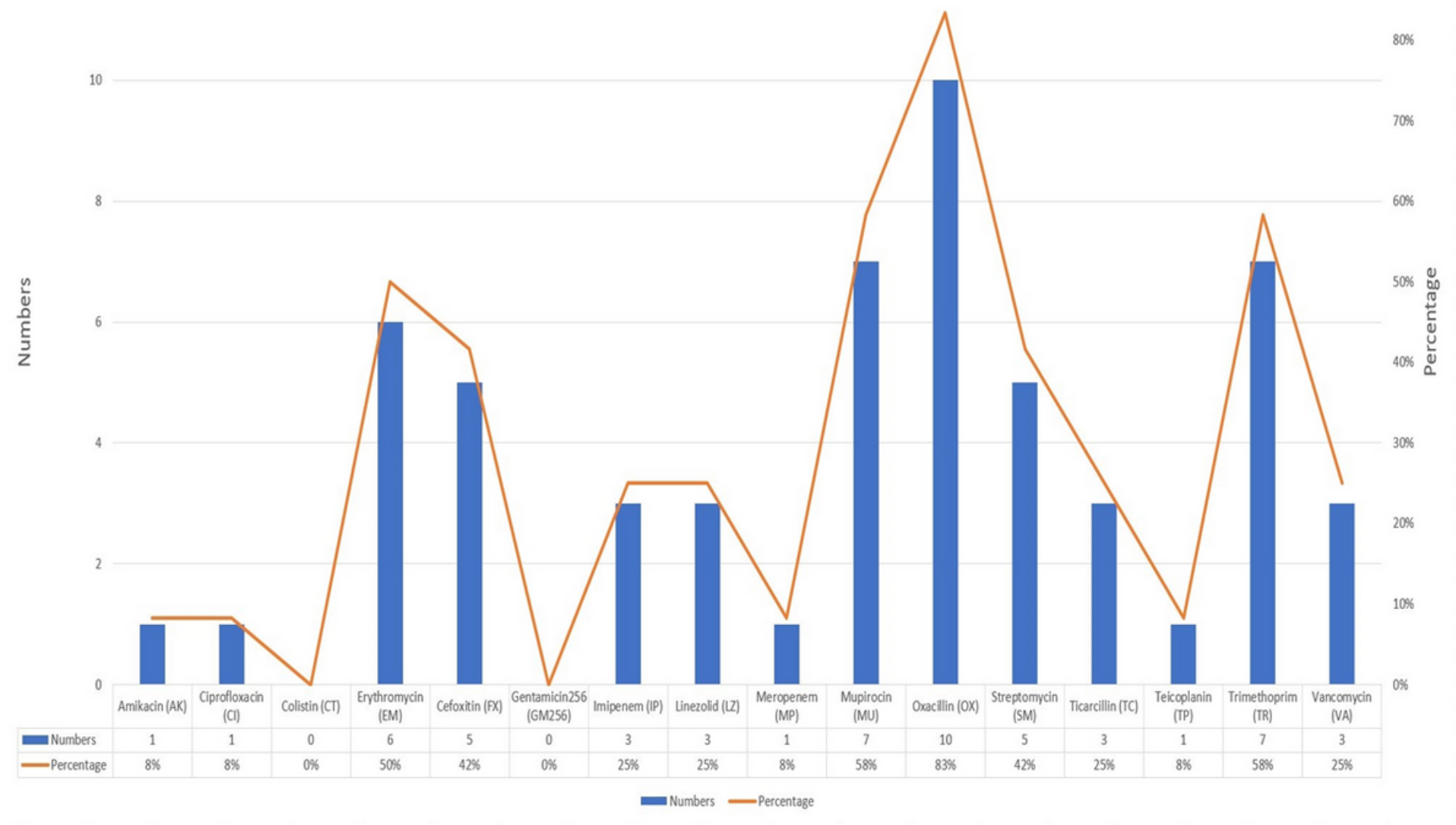

Figure 4

Both Solibacillus isronensis B3W22 isolates showed resistance to erythromycin, cefoxitin, imipenem, mupirocin, oxacillin, streptomycin, tetracycline, and trimethoprim (Figure.4). 


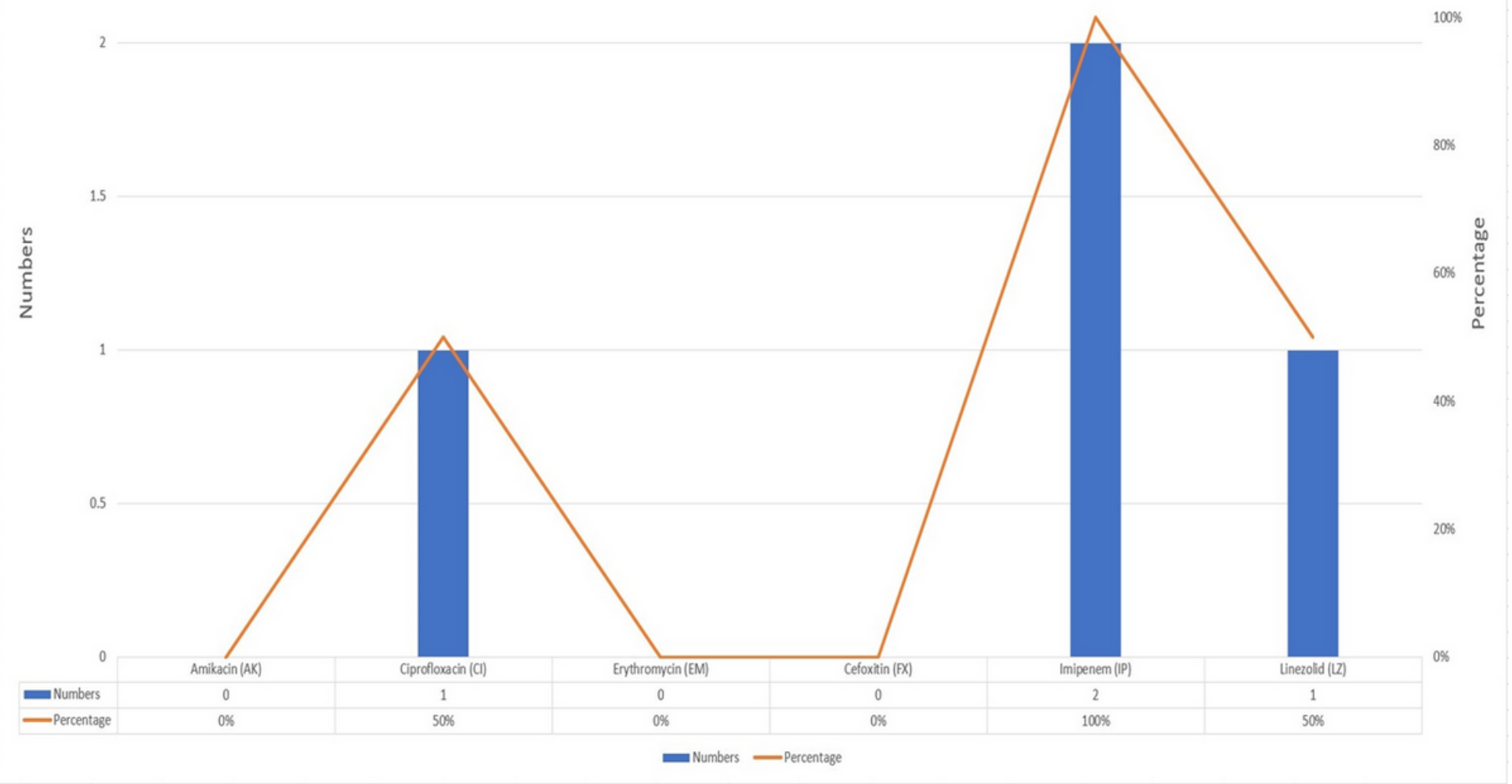

\section{Figure 5}

Two isolates of Pseudomonas spp. were obtained consisting of P. xanthomarina and P. xiamenensis DSM22326. Both showed resistance to Imipenem. P. xiamenensis exhibited multiple drug resistance pattern, including resistance to ciprofloxacin, linezolid and imipenem (Table 6) (Figure 5). 
20

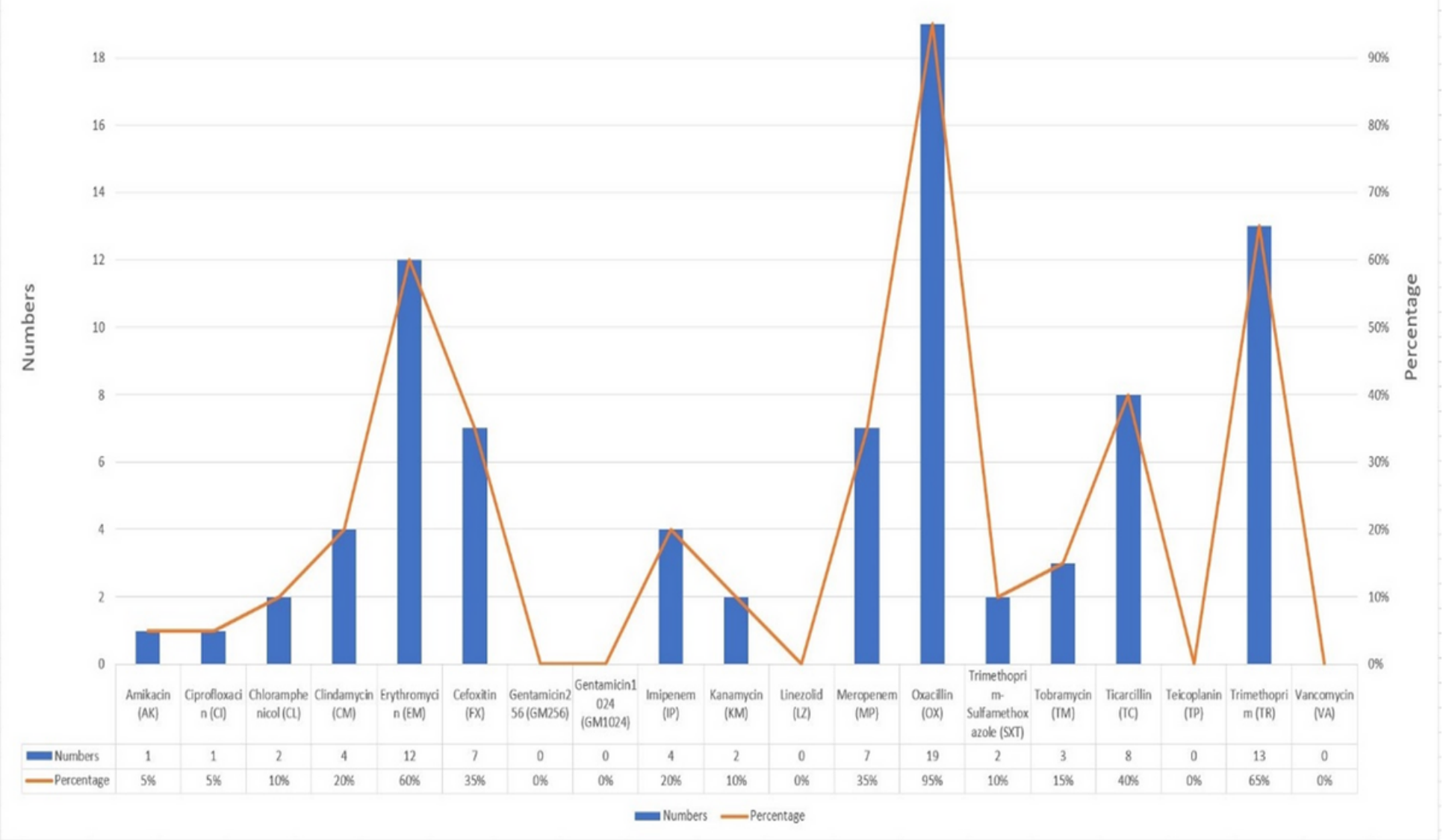

Figure 6

Additionally, there were $10 \%$ (2 out of 20 ) resistance to each of chloramphenicol, kanamycin, sulfamethoxazole-trimethoprim, and $5 \%$ ( 1 out of 20 ) resistance to each of amikacin and ciprofloxacin (Figure 6). 